Research Article

\title{
Experimental Study on Shock Wave Propagation of the Explosion in a Pipe with Holes by High-Speed Schlieren Method
}

\author{
Gang Zhang $\mathbb{D}$ \\ College of Energy Engineering, Xi'an University of Science and Technology, Xi'an 710054, China \\ Correspondence should be addressed to Gang Zhang; 19103077009@stu.xust.edu.cn
}

Received 16 June 2020; Revised 30 July 2020; Accepted 29 August 2020; Published 12 September 2020

Academic Editor: Chengzhi Shi

Copyright (C) 2020 Gang Zhang. This is an open access article distributed under the Creative Commons Attribution License, which permits unrestricted use, distribution, and reproduction in any medium, provided the original work is properly cited.

\begin{abstract}
The shock wave propagation of the explosion in a pipe with holes was studied by a high-speed schlieren experimental system. In the experiments, schlieren images in the explosion were recorded by a high-speed camera from parallel and perpendicular orientations, respectively, and the pressure in the air was measured by an overpressure test system. In parallel orientation, it is observed that the steel pipe blocks the propagation of blast gases, but it allows the propagation of shock waves with a symmetrical shape. In perpendicular orientation, oblique shock wave fronts were observed, indicating the propagation of explosion detonation along the charge. Shock wave velocity in the hole direction is larger than that in the nonhole direction, indicating the function of holes in controlling blast energy, that is, leading blast energy to hole direction. Furthermore, the function of holes is verified by overpressure measurements in which peak overpressure in the hole direction is $0.87 \mathrm{KPa}, 2.8$ times larger than that in the nonhole direction. Finally, the variation of pressure around the explosion in a pipe with holes was analyzed by numerical simulation, qualitatively agreeing with high-speed schlieren experiments.
\end{abstract}

\section{Introduction}

Blast has been widely applied in geotechnical engineering, such as rock roadway excavation, open-pit mining, and slope engineering [1]. In order to reduce the damage of the surrounding rock and improve its stability, directional fracture controlled blasting technology is introduced by changing the borehole or charge shape. Initially, Langefors and Kihlström [2] proposed a controlled blasting method by producing two notches in the borehole. Fourney et al. [3] used a split tube as the charge holder to produce controlled blast fractures. By using the slit charge holder and highspeed photography, Qiu Peng et al. [4, 5] studied the mechanism of the interaction between blast stress waves and controlled blast fractures. Yue et al. [6] used slit charge holder to study blast-induced crack propagation between two boreholes. Besides slit charge holder, He et al. [7] proposed a new directional blasting method by drilling holes in the charge holder and used this directional blasting method to cut roadway roof near the gob in coal mines.

Blast experiments are a useful approach to study blastinduced stress waves, gases, and cracks [8-10]. The most important is how to measure these parameters in a very short period. High-speed visualization of blast process by optical methods is a good way to study problems involving blast stress waves, blast gases, and cracks. Photoelasticity can visualize blast stress waves [11-13], while caustics method can visualize not only blast stress waves but also blast gases $[14,15]$. Both photoelasticity and caustics methods are able to obtain crack-tip stress intensity factors [11, 16, 17]. Specifically, to combine advantages of photoelasticity and caustics methods, Qiu Peng et al. [18] proposed a simultaneous optical measurement system combining photoelasticity and caustics methods, which is verified in the study on blast-induced dynamic fracture. However, photoelasticity and caustics methods meet difficulties in measurements near the borehole because data acquisition of isochromatic fringes in photoelasticity and shadow patterns in caustics method is very difficult in such an extreme condition. Digital image correlation (DIC) becomes a very effective method to study blast-induced stress waves $[19,20]$, but this method needs speckles or reference points on specimen surface and does not work well for blast gases. Schlieren method can visualize propagation of shock waves and blast-induced 
products near the borehole because this method is sensitive to gas dynamics in transparent media. Using schlieren method, Gao [21] studied the propagation process of shock waves and blast-induced products from a slit charge holder. In addition, by schlieren method and numerical simulation, Yang and Zuo [22] also studied the directional fracture mechanism of slit charge blasting. These blast experiments using schlieren method verify that this optical method is a useful approach to study shock waves and blast products near the borehole.

A large number of researchers used optical methods to study slit charge blasting method; however, another important directional blasting method, that is, a charge holder with holes, gets less attention. A charge holder with holes is practically useful in cutting roadway roof in coal mines, but its mechanism of shock wave propagation is not specific, partly because shock wave propagation cannot be visualized in the rock mass, partly due to limitations in high-speed measurements.

In this paper, the mechanism of shock wave propagation of the charge in a pipe with holes was studied by high-speed schlieren method, overpressure test, and numerical simulation. Experimental preparations are presented in Section 2. In Section 3, high-speed schlieren images were recorded in parallel and perpendicular orientations, respectively, and then shock wave velocity was analyzed. Additionally, overpressure in air around the explosion was measured in hole and nonhole directions. Finally, numerical simulation was conducted for a better understanding of shock wave propagation of the charge in a pipe with holes.

\section{Experimental Preparations}

2.1. High-Speed Schlieren Experimental System. The highspeed schlieren experimental system is shown in Figure 1, which consists of four parts: light source area, lenses combination, reaction area, and acquisition area. The light source area is composed of a laser, beam expander, knifeedge, and plane mirror, and the lenses combination consists of two field lenses (concave lens). The reaction area is located between two filed lenses and covered in an iron box for securities. The acquisition area is composed of a planar mirror, a knife-edge, and a high-speed camera (Photron SA5). The frequency of the camera was 100,000 frames per second in the present experiments.

The propagation process of laser light rays is described here. The laser light first passes through the knife-edge in front of the plane mirror, and then the plane mirror and the field lens 1 reflect the parallel light to make it pass through the reaction area. Subsequently, the laser light reflects at field lens 2 and the second plane mirror and passes through the second knife-edge. Finally, schlieren images of the reaction area are recorded by the high-speed camera.

2.2. Charge Holder of a Pipe with Holes. Figure 2 shows the charge holder of a stainless steel pipe with a length of $100 \mathrm{~mm}$. The outer and inner diameters of the pipe are $14 \mathrm{~mm}$ and $11 \mathrm{~mm}$, respectively; thus, pipe wall thickness is
$3 \mathrm{~mm}$. The holes for concentrating blasting energy were drilled along the paper wall, with hole diameter and space equal to $2 \mathrm{~mm}$ and $3 \mathrm{~mm}$, respectively.

The explosive used in the experiments was lead azide. A column charge with a diameter of $6 \mathrm{~mm}$ was inserted into the stainless steel pipe with holes. The charge effective charge length is $80 \mathrm{~mm}$ and charge quantity is $800 \mathrm{mg}$. Two stem areas are located at two ends of the charge, each with a length of $10 \mathrm{~mm}$. A pair of twisted copper wires were used to initiate the explosive, and the initiation point was located at the central position of the column charge.

An iron box was used to cover the charge holder and protect the experimental system and the people who conduct blasting experiments. A large piece of sponge was attached to the inner wall of the iron box to absorb the blasting energy. The observation window was equipped with a transparent PMMA plate to avoid the damage caused by the blast-induced flyers and gases to the equipment, especially to the high-speed camera.

\subsection{Parallel and Perpendicular Orientations of the Charge} Holder. For a comprehensive understanding of shock wave propagation in a three-dimensional view, the shock wave propagation process of the charge in a stainless steel pipe with holes was recorded by the high-speed camera from two different orientations: parallel and perpendicular orientations, as shown in Figure 3. For parallel orientation, the axial direction of the charge pipe is parallel to the light direction while, for perpendicular orientation, the axial direction of the charge pipe is perpendicular to the light direction.

\section{Experimental Results and Discussion}

3.1. High-Speed Schlieren Images. Figure 4 shows the propagation process of shock waves and blast gases produced by the charge in parallel orientation where the charge direction is parallel to laser light. Laser light direction is perpendicular to the page. Firstly, obvious deflagration phenomena can be observed in the process of detonation, as shown in a period of $10 \sim 20 \mu \mathrm{s}$. It should be noted that the schlieren experimental system cannot observe the detonation process occurring in the pipe; thus the propagation of detonation waves in the pipe was not studied here. Additionally, the process of the shock waves propagating out of the field of view is not listed here. Due to limitations in blast experiments, the interaction between holes is not clearly visualized because blast-induced gases influence the highspeed visualization. In the future, the interaction between holes will be investigated by improving the high-speed recording system.

In parallel orientation in Figure 4, shock waves and the blast gases propagate in a highly symmetrical shape. Shock waves caused by continuous detonation of the charge in the pipe first run out from holes at $10 \mu \mathrm{s}$, followed by blast gases. Shock wave front forms an envelope to surround blast gases. The distance between shock waves front and the blast gases increased gradually with the propagation of shock waves. In a period of $80 \sim 100 \mu \mathrm{s}$, it is observed that the steel pipe 


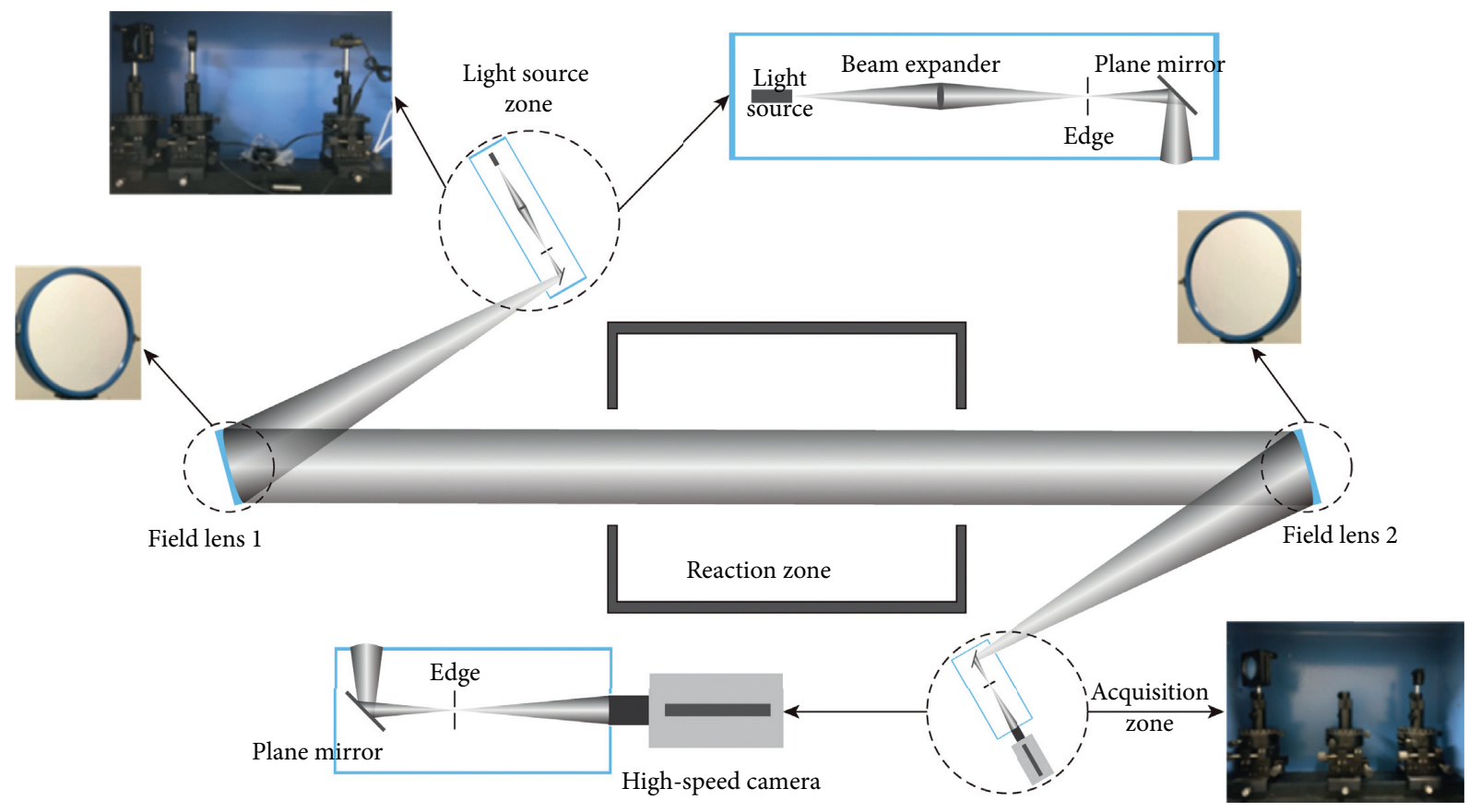

Figure 1: Schematic of the high-speed schlieren system.

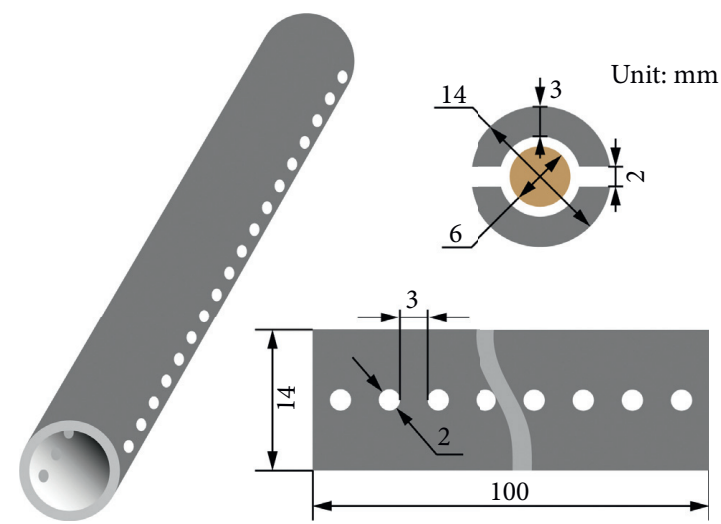

(a)

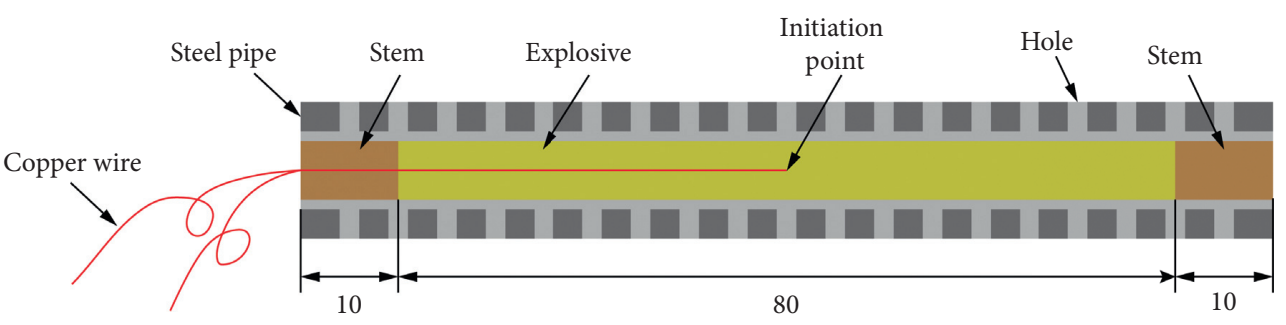

(b)

FIGURE 2: Schematic of the charge holder of a pipe with holes.

blocks the propagation of blast gases in the top and bottom direction, but it allows the propagation of shock waves, which is marked by a dot circle in Figure 4. Based on this consideration, it is easy to distinguish shock wave front and blast gases. Holes along the pipe change the propagation of shock waves and blast gases. The shape of shock waves and blast gases is the superposition of two circles while, without the presence of the holes, shock waves are a circle shape. In addition, holes provide a preferred way for blast products. It is observed that blast products run out of the charge holder from holes in two sides of the pipe, which are marked by the arrows in Figure 4. 


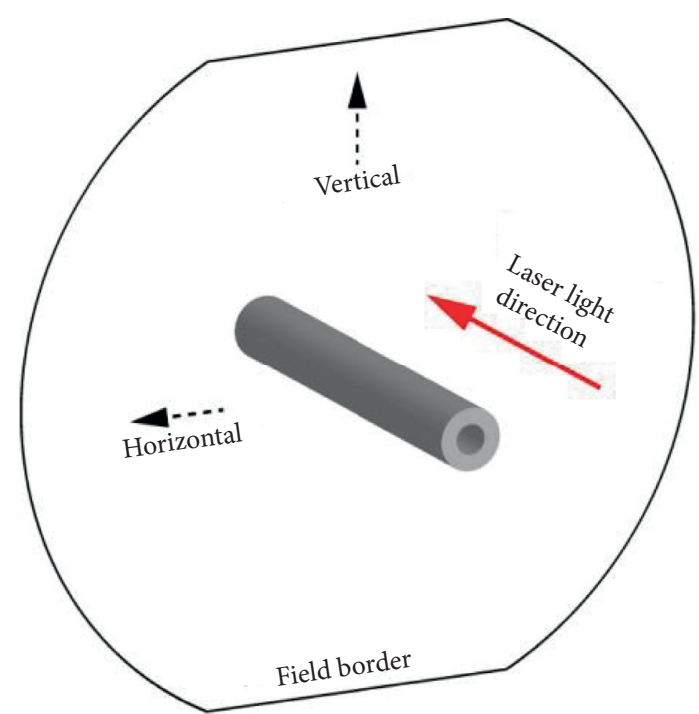

(a)

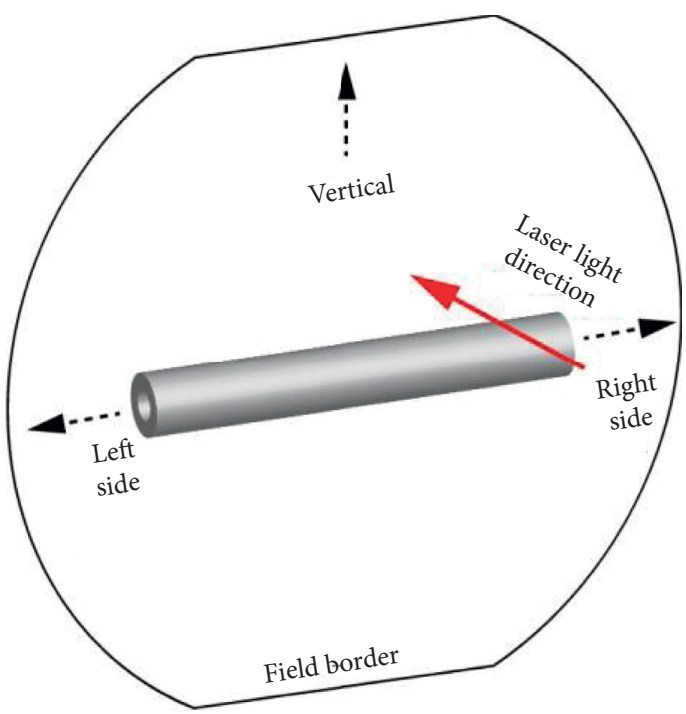

(b)

FIGURE 3: Schematic of the (a) parallel and (b) perpendicular orientations.

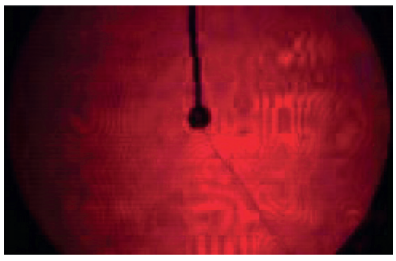

(a)

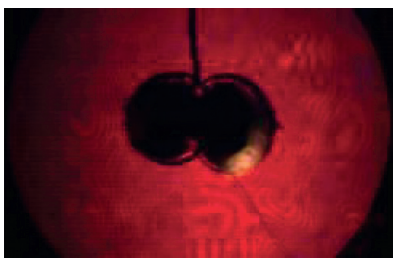

(e)

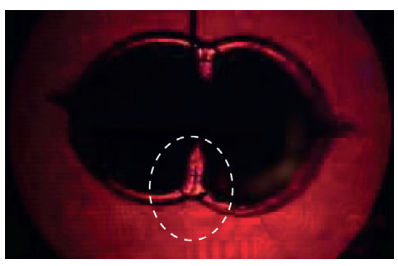

(i)

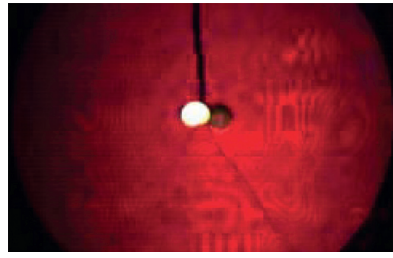

(b)

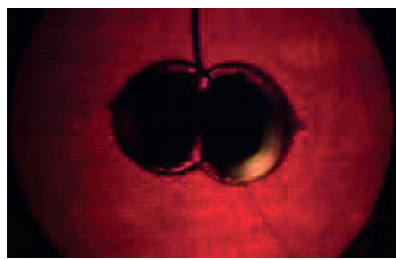

(f)

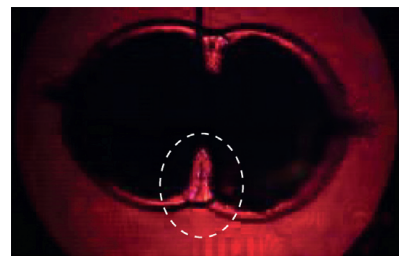

(j)

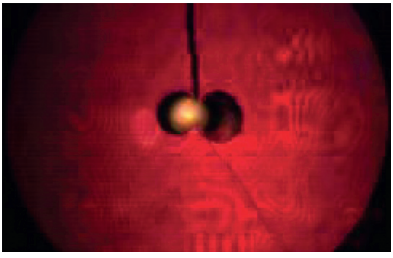

(c)

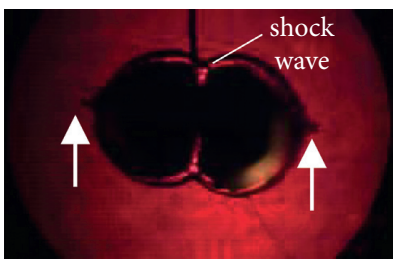

(g)

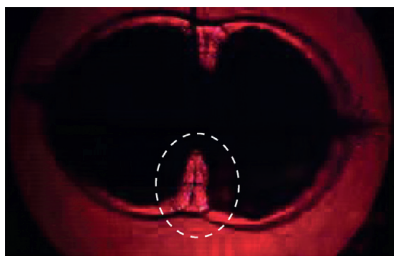

(k)

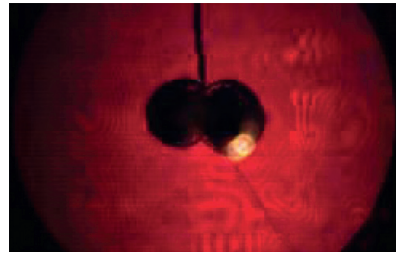

(d)

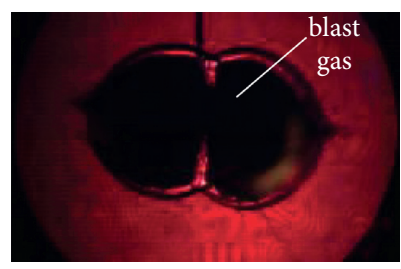

(h)

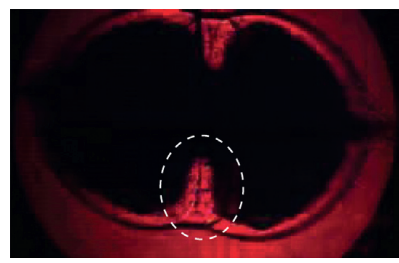

(1)

FIGURE 4: High-speed schlieren images for parallel orientation of the charge (parallel to laser light). (a) $0 \mu \mathrm{s}$, (b) $10 \mu \mathrm{s}$, (c) $20 \mu \mathrm{s}$, (d) $30 \mu \mathrm{s}$, (e) $40 \mu \mathrm{s}$, (f) $50 \mu \mathrm{s}$, (g) $60 \mu \mathrm{s}$, (h) $70 \mu \mathrm{s}$, (i) $80 \mu \mathrm{s}$, (j) $90 \mu \mathrm{s}$, (k) $100 \mu \mathrm{s}$, and (l) $110 \mu \mathrm{s}$.

Figure 5 shows the propagation process of shock waves and blast gases produced by the charge in perpendicular orientation where the charge direction is perpendicular to laser light. Laser light direction is perpendicular to the page. The initiation point of the charge is located at the right of the center of the pipe, which is implied at $10 \mu \mathrm{s}$, and oblique shock wave fronts are symmetrical by the pipe axis and propagate towards two ends of the charge at $20 \sim 30 \mu$ s. The oblique shock wave fronts indicate that detonation wave speed in the charge is faster than that of shock waves in the air. At $40 \mu \mathrm{s}$, shock waves and blast gases rush out of the right end of the pipe, making an influence on the shape of the 


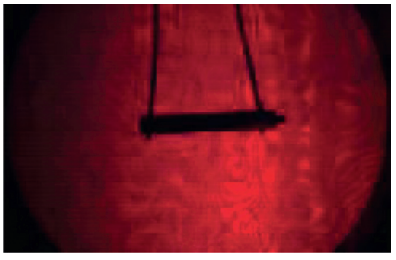

(a)

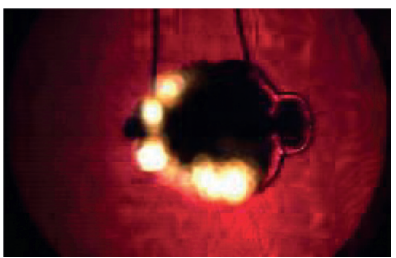

(e)

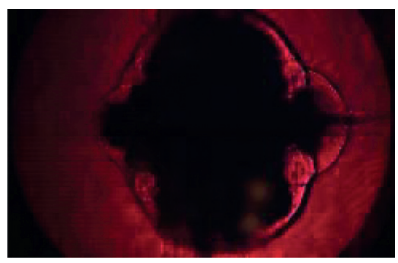

(i)

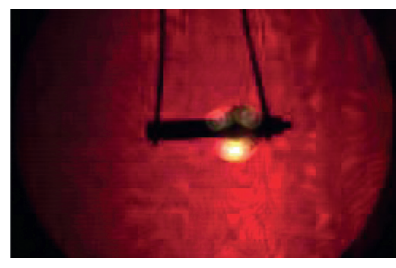

(b)

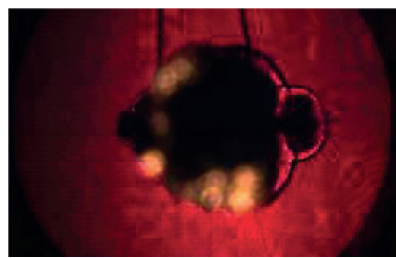

(f)

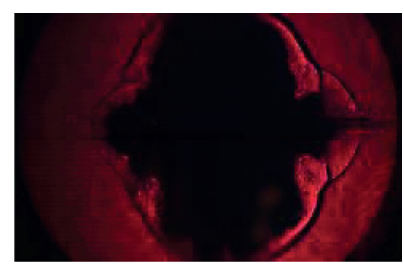

(j)

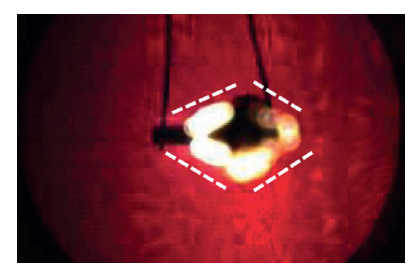

(c)

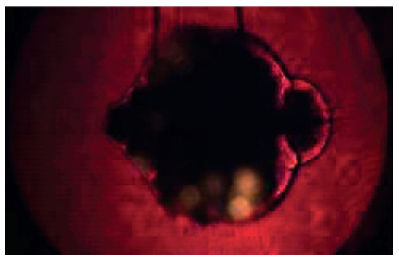

(g)

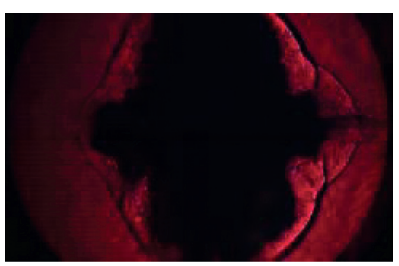

(k)

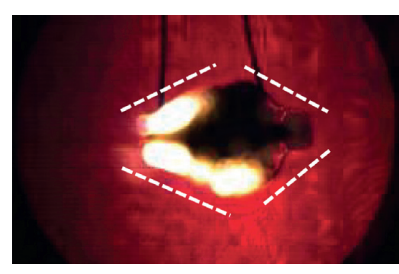

(d)

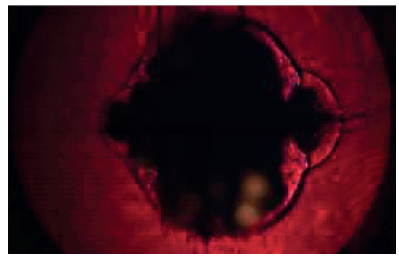

(h)

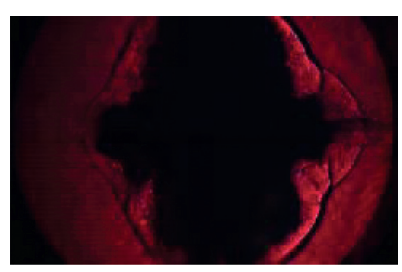

(1)

FIGURE 5: High-speed schlieren images for perpendicular orientation of the charge (perpendicular to laser light). (a) $0 \mu \mathrm{s}$, (b) $10 \mu \mathrm{s}$, (c) $20 \mu \mathrm{s}$, (d) $30 \mu \mathrm{s}$, (e) $40 \mu \mathrm{s}$, (f) $50 \mu \mathrm{s}$, (g) $60 \mu \mathrm{s}$, (h) $70 \mu \mathrm{s}$, (i) $80 \mu \mathrm{s},(j) 90 \mu \mathrm{s},(\mathrm{k}) 100 \mu \mathrm{s}$, and (l) $110 \mu \mathrm{s}$.

whole shock waves and blast gases. Also, some blast products fly out from the right end of the pipe. The situation of the left end is similar to the right one. Therefore, the quality of the stemming is of significant importance for controlled blasting. After $60 \mu \mathrm{s}$, the shape of shock waves and blast gases nearly remains the same while the size increases steadily.

\subsection{Shock Wave Front Propagation Velocity. Figure 6 shows} the velocity of the shock wave front in different orientations. In each orientation, the horizontal and vertical velocities of shock wave front were measured. In the parallel orientation, the whole shock wave propagation process presents a symmetrical shape centered by the pipe axis, and the propagation of shock waves on both sides of the pipe tends to be the same. Thus, the horizontal velocity of shock waves on one side was measured. In the perpendicular orientation, the propagation of shock waves in the horizontal left and right directions was measured, respectively, while the propagation of shock waves in the vertical direction was only measured on the top side, due to the symmetrical wave front.

In Figure 6(a), it is obvious that horizontal velocity is larger than that in the vertical direction because the holes in the pipe allow shock waves to run out preferentially in the horizontal direction, especially at $10 \sim 20 \mu$ s just after the initiation of the charge. After $20 \mu \mathrm{s}$, both horizontal and vertical velocities vary around $750 \mathrm{~m} / \mathrm{s}$. The difference of shock wave velocity in the horizontal and vertical directions indicates the function of holes in controlling blast energy, that is, leading blast energy to the hole direction.

In Figure 6(b), it is found that shock wave velocity in the right side of the charge has a sharp rise in $10 \sim 30 \mu$ s, much larger than that on the left side. This difference can be reflected in Figure 5 where shock wave front in the right side runs out prior to that on the left side mainly because stemming in the right side is looser than that on the left side of the charge. After $40 \mu \mathrm{s}$, both right and left sides have a similar velocity variation trend and fluctuate around $350 \mathrm{~m} /$ s. The vertical velocity of shock waves remains around $750 \mathrm{~m} / \mathrm{s}$ after $30 \mu \mathrm{s}$, agreeing with that in Figure 6(a). It should be noted that after $80 \mu$ s the shock wave front runs out of the field of view; thus, its velocity cannot be measured in vertical direction.

3.3. Overpressure Measurement. To better understand the function of holes in the pipe, the overpressure in the air around the explosion was measured. The overpressure test system is composed of Teledyne Lecroy HDO 4034 electronic oscilloscope, Sinocera YE5853 A charge amplifier and CY-YD-202 pressure sensor, shown in Figure 7(a). In order to avoid unnecessary damage to the sensor in the process of explosion, the distance between the sensor and the explosion center was set to $200 \mathrm{~mm}$. Two sensors correspond to horizontal and vertical directions, respectively, illustrated in Figure 7(b).

In the instant of detonation, a large number of high temperature and high pressure gases will be generated in the pipe and then rapidly expand outward from holes and form shock waves. Subsequently, expanding blast gases run out from the holes and push the air around the pipe. Shock waves decay with distance from the pipe, and the velocity of gases also decreases until blast gases no longer expand. The positive pressure zone has a prominent influence on the air 


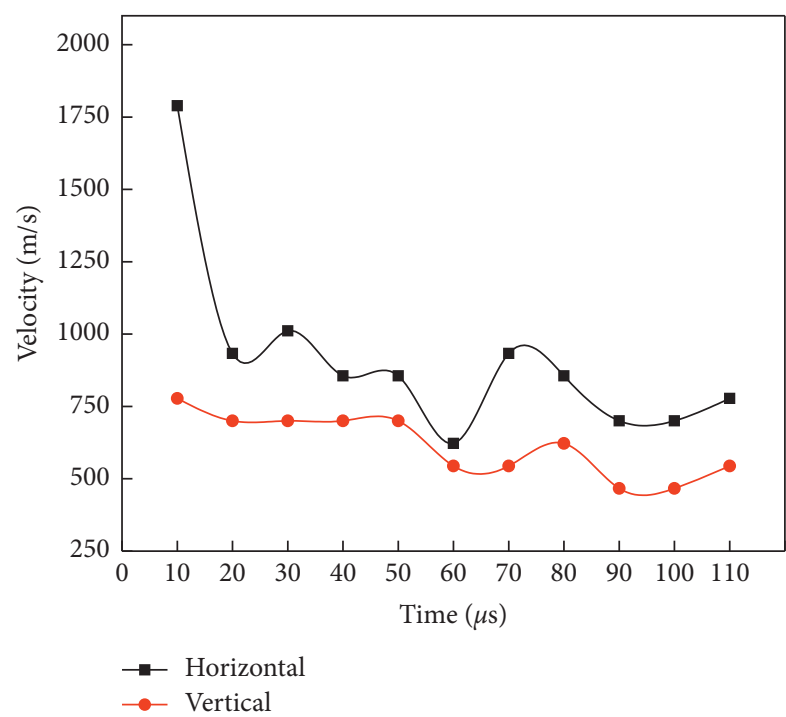

(a)

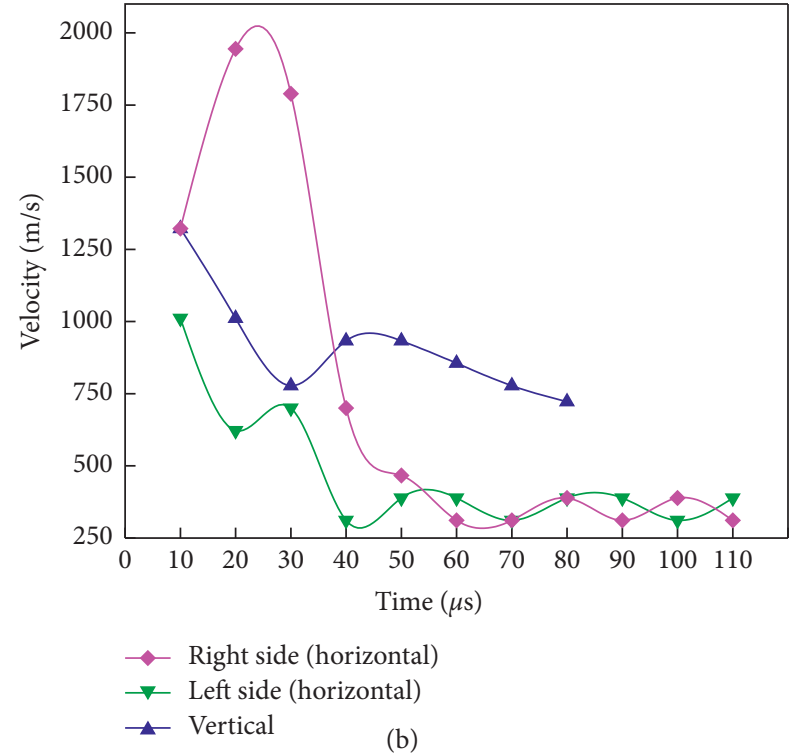

(b)

FIGURE 6: Shock wave front velocity in (a) parallel and (b) perpendicular orientations of the charge.

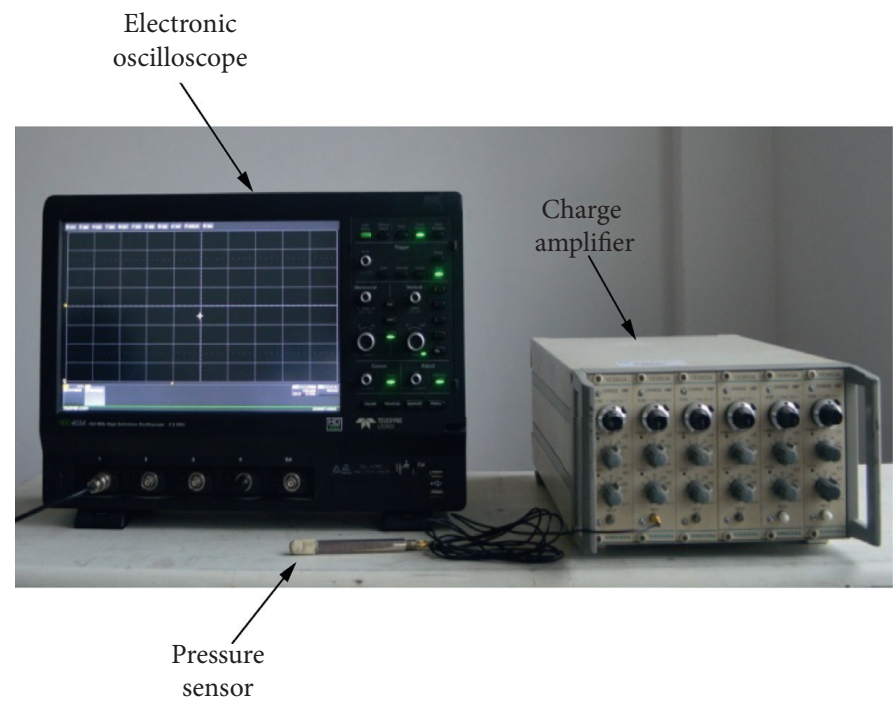

(a)

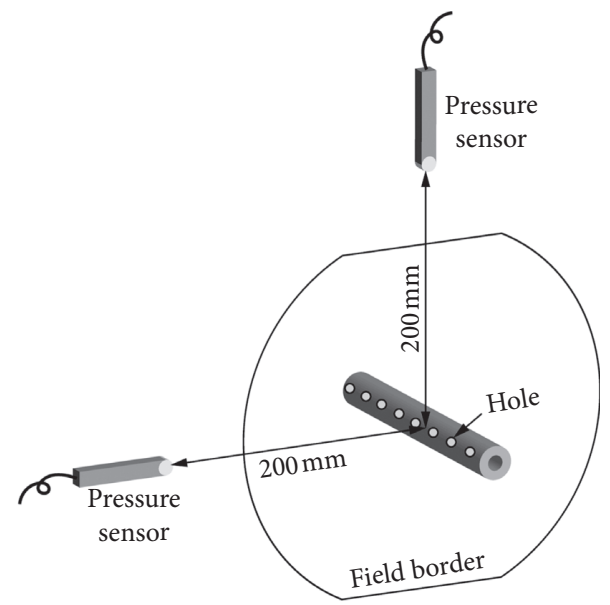

(b)

Figure 7: (a) Photo of overpressure measuring device. (b) Schematic of the overpressure test system.

around the pipe, followed by the negative pressure zone, which is shown in Figure 8.

Figure 8 shows the history of overpressure with time. Because the overpressure sensors were $200 \mathrm{~mm}$ far from the detonation center, the pressure was measured at $\mathrm{kPa}$ level. The shock wave front in the hole direction presses the pressure sensor at $243 \mu \mathrm{s}$, resulting in a very sharp rise up to a peak value of $0.87 \mathrm{kPa}$. Later, at $490 \mu$ s, the shock front in the nonhole direction pressures the pressure sensor, resulting in a smaller rise with a peak value of $0.31 \mathrm{kPa}$. The peak overpressure in the hole direction is 2.8 times larger than that in the nonhole direction, and the time to reach the peak value in the hole direction is only $1 / 2$ of that in the nonhole direction. The peak overpressure difference in the hole and nonhole directions indicate that holes in the pipe control blast energy, making energy concentration in the hole direction. Compared with Figure 4, the peak overpressure of $0.87 \mathrm{kPa}$ in the hole direction corresponds to blast products in the hole direction, and the peak overpressure of $0.31 \mathrm{kPa}$ in the nonhole direction corresponds to blast products in the nonhole direction. After peak overpressure, pressure fluctuation appears in both the hole and nonhole directions. It is found that pressure fluctuation value in the hole direction is larger than that in the nonhole 


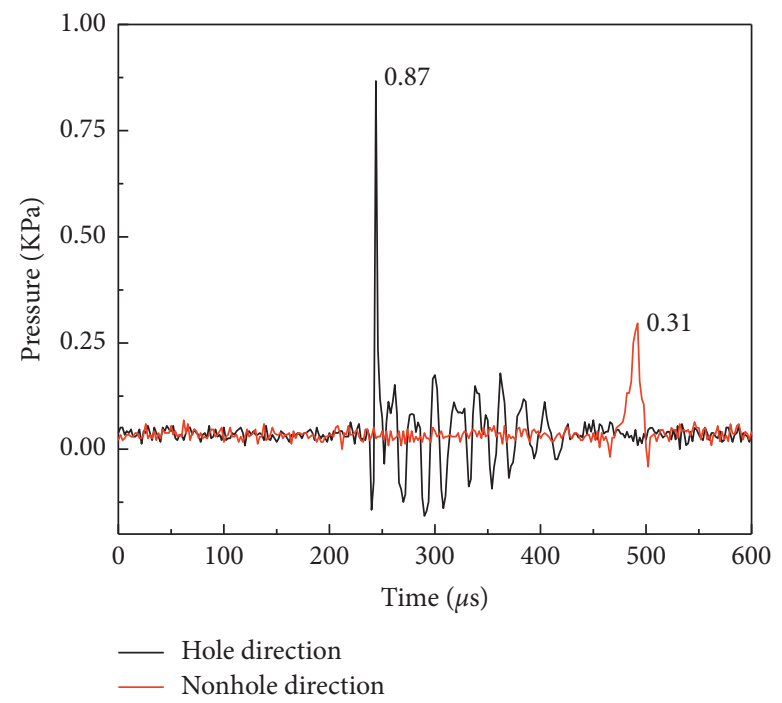

FIgURE 8: Overpressure results from two directions (hole and nonhole directions).

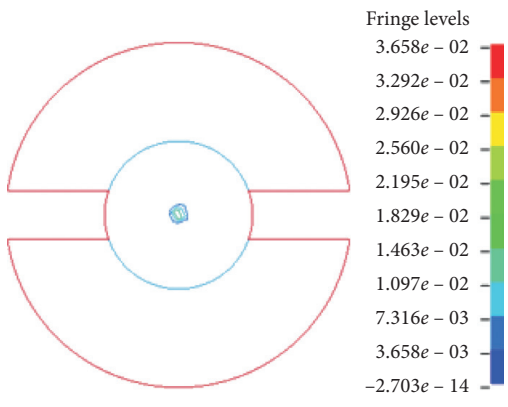

(a)

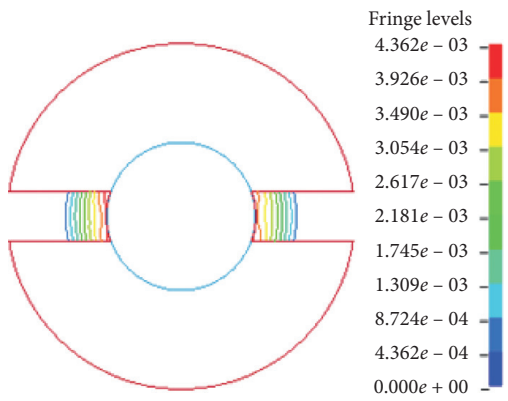

(c)

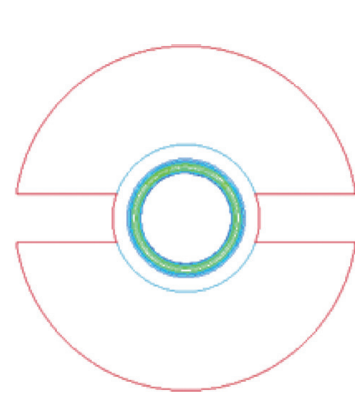

Fringe level

$1.183 e-01$

$1.064 e-01=$

$9.462 e-02-$

$8.279 e-02$

$7.096 e-02$

$5.914 e-02-$

$4.731 e-02$

$3.548 e-02=$

$2.365 e-02$

$1.183 e-02$

(b)

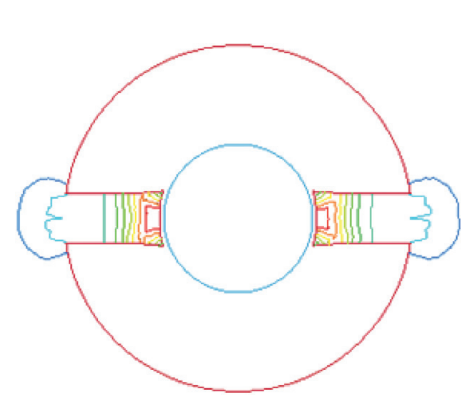

iningevels $8.380 e-03$

$7.542 e-03$

$6.704 e-03-$

$5.866 e-03=$

$5.028 e-03$

$4.190 e-03-$

$3.352 e-03$

$2.514 e-03-$

$1.676 e-03$

$8.380 e-04$

$0.000 e+00$

(d)

Fringe levels

$5.204 e-03-$

$4.684 e-03$

$4.163 e-03-$

$3.643 e-03=$

$3.123 e-03=$

$2.602 e-03=$

$2.082 e-03=$

$1.561 e-03$

$1.041 e-03$

$5.204 e-04$

$0.000 e+00$

(e)

Figure 9: Continued. 


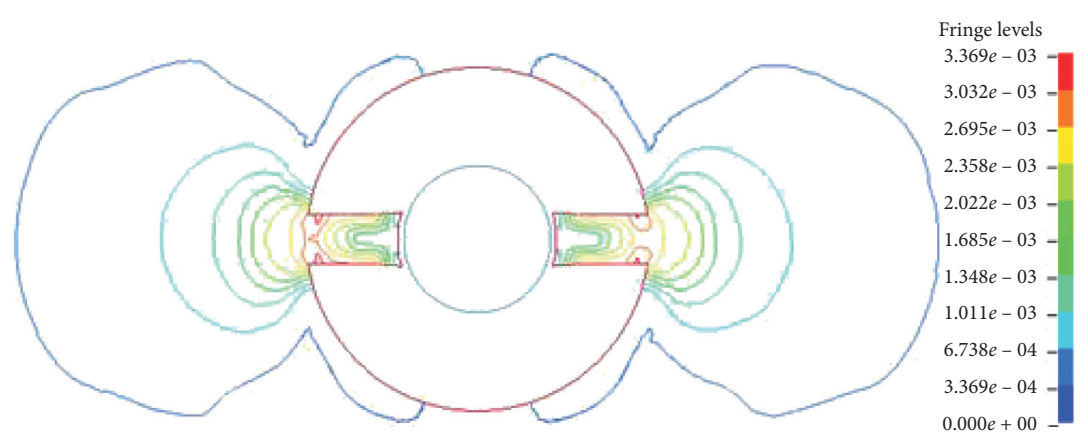

(f)

Figure 9: The pressure fluctuation process of numerical results. (a) $0.05 \mu \mathrm{s}$, (b) $0.36 \mu \mathrm{s}$, (c) $0.85 \mu \mathrm{s}$, (d) $2.05 \mu \mathrm{s}$, (e) $2.05 \mu \mathrm{s}$, (f) $2.89 \mu \mathrm{s}$, and (g) $4.85 \mu \mathrm{s}$.

direction, and the duration of the fluctuation is longer in the hole direction. After fluctuation and attention, the pressure value in air returns back to $0 \mathrm{kPa}$ in both the hole and nonhole directions.

\section{Numerical Verification}

Considering that the pressure field near the explosion cannot be observed clearly by schlieren experiments, ANSYS/LSDYNA finite element program was used to simulate the whole pressure field near the charge. The numerical simulation models were all SOLID164 units. The size and structure of pipe models were the same as those in schlieren experiments. Lagrange elements were used for the pipe model, and ALE elements were used for explosives and air. The fluid-solid coupled numerical solution method was adopted. EOS_LINEAR_POLYNOMIAL equation of state was used for air and JWL equation of state was used for the explosive. The explosives were made of lead azide with a density of $2 \mathrm{~g} / \mathrm{cm}^{3}$, a detonation heat of $1.53 \mathrm{~kJ} / \mathrm{g}(367 \mathrm{cal} / \mathrm{g})$, and a detonation velocity of $4070 \mathrm{~m} / \mathrm{s}$.

Figure 9 shows the pressure contour of the explosion at the cross section of the charge in pipe with two holes. The shock waves propagate outward rapidly at $0.36 \mu$ s and then run outward along the holes. The pressure influence area expands rapidly at $2.89 \mu \mathrm{s}$, but the peak pressure decreases due to gas expansion. Expanding gases first influence the area in the hole direction and then go to the nonhole direction, which agrees with the propagation of blast-induced products in high-speed schlieren images in Figure 4. For points with the same distance from the explosion center, the pressure value in the hole direction is obviously larger than that in the nonhole direction, which is similar to overpressure testing results in Figure 8 $(0.87 \mathrm{kPa}$ in the hole direction and $0.31 \mathrm{kPa}$ in the nonhole direction). And the pressure boundary in the hole direction is also larger than that in the nonhole direction. The shape of pressure field in Figure 8 is highly symmetrical, which is consistent with high-speed schlieren images in blast experiments.

This paper mainly focuses on blasting experiments. Numerical simulation in this paper was carried out in a twodimensional way. Some previous papers provide good references in numerical simulations associated with pipes
[23-25]. In the future, three-dimensional numerical methods will be used to understand the mechanism of shock wave propagation of the explosion in a pipe with holes, and the interaction of shock waves and blast gases between holes will be further investigated.

\section{Conclusions}

The high-speed schlieren photography, overpressure measurement, and numerical simulation were applied to study shock wave propagation of the charge in a pipe with holes, and the following conclusions are obtained:

(1) Shock wave propagation of the charge in a pipe with holes was recorded by the high-speed camera from two different orientations: parallel and perpendicular orientations. In parallel orientation, it is observed that the steel pipe blocks the propagation of blast gases in the top and bottom side, but it allows the propagation of shock waves with a symmetrical shape. In perpendicular orientation, oblique shock wave fronts were observed, indicating the propagation of explosion detonation in the column charge.

(2) Measurements of high-speed schlieren images show that the shock wave velocity in the hole direction is larger than that in nonhole direction, verifying the function of holes in controlling blast energy, that is, leading blast energy to the hole direction.

(3) Measurements of overpressure in air around the explosion show that peak overpressure in the hole direction is $0.87 \mathrm{kPa}, 2.8$ times larger than that in the nonhole direction, and the time to reach the peak value in the hole direction is only $1 / 2$ that in the nonhole direction. The difference in overpressure again verifies the function of holes in the pipe.

(4) The variation of pressure around the explosion in a pipe with holes is analyzed by the numerical simulation, qualitatively agreeing with high-speed schlieren experiments.

\section{Data Availability}

The data required to reproduce these findings is available from the author upon request. 


\section{Conflicts of Interest}

The author declares that there is no competing financial interest or personal relationship that influences the work reported in this paper.

\section{References}

[1] M. Saharan, H. Mitri, and J. Jethwa, "Rock fracturing by explosive energy: review of state-of-the-art," Fragblast, vol. 10, no. 1-2, pp. 61-81, 2006.

[2] U. Langefors and B. Kihlström, The Modern Technique of Rock Blasting, Wiley, Hoboken, NY, USA, 1963.

[3] W. L. Fourney, J. W. Dally, and D. C. Holloway, "Controlled blasting with ligamented charge holders," International Journal of Rock Mechanics and Mining Sciences \& Geomechanics Abstracts, vol. 15, no. 3, pp. 121-129, 1978.

[4] P. Qiu, Z. Yue, and R. Yang, "Mode I stress intensity factors measurements in PMMA by caustics method: a comparison between low and high loading rate conditions," Polymer Testing, vol. 76, pp. 273-285, 2019.

[5] P. Qiu, Z. Yue, R. Yang, and J. C. Li, "Effects of vertical and horizontal reflected blast stress waves on running cracks by caustics method," Engineering Fracture Mechanics, vol. 212, pp. 164-179, 2019.

[6] Z. W. Yue, L. Y. Yang, and Y. B. Wang, "Experimental study of crack propagation in polymethyl methacrylate material with double holes under the directional controlled blasting," $\mathrm{Fa}$ tigue \& Fracture of Engineering Materials \& Structures, vol. 36, no. 8, pp. 827-833, 2013.

[7] M. He, W. Cao, R. Shan, and S. Wang, "New blasting technology-bilateral cumulative tensile explosion," Chinese Journal of Rock Mechanics and Engineering, vol. 22, no. 12, pp. 2047-2051, 2003.

[8] C. Liu, Y. Lu, B. Xia, and P. Yu, "Directional fracturing by slotting-blasting-caused stress wave form changes," International Journal of Impact Engineering, vol. 129, pp. 141-151, 2019.

[9] D. Wan, Z. Zhu, R. Liu, B. Liu, and J. Li, "Measuring method of dynamic fracture toughness of mode I crack under blasting using a rectangle specimen with a crack and edge notches," International Journal of Rock Mechanics and Mining Sciences, vol. 123, Article ID 104104, 2019.

[10] D. Xiao, Z. Zhu, R. Hu, and L. Lang, "Study of testing method for dynamic initiation toughness of sandstone under blasting loading," Shock and Vibration, vol. 2018, Article ID 1043298, 13 pages, 2018.

[11] J. W. Dally, "Dynamic photoelastic studies of fracture," Experimental Mechanics, vol. 19, no. 10, pp. 349-361, 1979.

[12] R. Yang, C. Chen, P. Xu, C. Ding, and Z. Zhang, "Experimental investigation of obliquely incident blast wave effect on deflection of running cracks," Journal of Testing and Evaluation, vol. 49, no. 2, 2019.

[13] R. Yang, C. Chen, Z. Yue, X. Xang, P. Xu, and C. Ding, "Dynamic photoelastic investigation of interaction of normal incidence blasting stress waves with running cracks," Journal of China Coal Society, vol. 43, no. 1, pp. 87-94, 2018.

[14] P. Qiu, Z. Yue, Y. Ju, and J. Zhao, "Characterizing dynamic crack-tip stress distribution and evolution under blast gases and reflected stress waves by caustics method," Theoretical and Applied Fracture Mechanics, vol. 108, Article ID 102632, 2020.

[15] Z. Yue, P. Qiu, R. Yang, S. Zhang, K. Yuan, and Z. Li, "Stress analysis of the interaction of a running crack and blasting waves by caustics method," Engineering Fracture Mechanics, vol. 184, pp. 339-351, 2017.

[16] X. F. Yao and W. Xu, "Recent application of caustics on experimental dynamic fracture studies," Fatigue \& Fracture of Engineering Materials \& Structures, vol. 34, no. 6, pp. 448459, 2011.

[17] W. Liu, Z. Yue, and G. Yang, "Experimental investigation of a circumferential crack in a PMMA cylindrical shell using caustics," Polymer Testing, vol. 79, Article ID 106086, 2019.

[18] P. Qiu, Z. Yue, S. Zhang, and Z. Li, “Anin situsimultaneous measurement system combining photoelasticity and caustics methods for blast-induced dynamic fracture," Review of Scientific Instruments, vol. 88, no. 11, Article ID 115113, 2017.

[19] R. Yang, S. Fang, A. Yang, H. Xie, and L. Yang, "In situ stress effects on smooth blasting: model test and analysis," Shock and Vibration, vol. 2020, 14 pages, 2020.

[20] H. Schreier, J.-J. Orteu, and M. A. Sutton, Image Correlation for Shape, Motion and Deformation Measurements: Basic Concepts, Theory and Applications, Springer, Berlin, Germany, 2009.

[21] X. Gao, Detonation Shock Dynamic Behavior of Split-Tube Charge Holder, China University of Mining \& Technology, Beijing, China, 2013.

[22] R. Yang and J. Zuo, "Experimental study on directional fracture blasting of cutting seam cartridge," Shock and Vibration, vol. 2019, Article ID 1085921, 11 pages, 2019.

[23] E. Liu, W. Li, H. Cai, and S. Peng, "Formation mechanism of trailing oil in product oil pipeline," Processes, vol. 7, no. 1, 2019.

[24] Z. Su, E. Liu, Y. Xu, P. Xie, C. Shang, and Q. Zhu, "Flow field and noise characteristics of manifold in natural gas transportation station," Oil \& Gas Science and Technology-Revue D'IFP Energies Nouvelles, vol. 74, 70 pages, 2019.

[25] S. Peng, Q. Chen, C. Zheng, and E. Liu, "Analysis of particle deposition in a new-type rectifying plate system during shale gas extraction," Energy Science \& Engineering, vol. 8, no. 3, pp. 702-717, 2020. 